\title{
ENVELOPING, FRICTIONLESS, PLANAR GRASPING
}

\author{
Jeffrey C. Trinkle \\ GRASP Laboratory \\ Department of Systems Engineering
}

\author{
Jacob M. Abel \\ Department of Mechanical Engineering \\ and Applied Mechanics
}

\author{
Richard P. Paul \\ GRASP Laboratory \\ Department of Computer and \\ Information Science
}

University of Pennsylvania, Philadelphia, PA 19104

\section{ABSTRACT}

Grasping by a two-dimensional hand comprised of a palm and two hinged fingers is studied. The mathematics of frictionless grasping is presented and used in the development of a planner/simulator. The simulator computes the motion of the object using an active constraint set method and assuming exact knowledge of the physical properties of the polygonal object, hand, and support.

\section{Introduction}

Flexible manufacturing workcells typically contain a robot arm and many expensive, special-purpose, end-of-arm tools. The potential for cost and time savings through the use of a general purpose hand has fueled much research in the last ten years on designing, analyzing, and programming articulated mechanical hands $[3,6,8,10,11,12,18,21]$. Most studies have proceeded under the assumption that contact fricton forces will be large enough to keep the object from sliding on the fingers. In contrast, this paper is concerned with the mechanical analysis of "hands" when the friction forces are not large enough to prevent sliding. The results are applied to planning grasps using the surfaces of the hand, not just the finger tips.

\subsection{Previous Work}

The potential for grasping and manipulating a wide variety of objects with a single end effector has encouraged research in grasp mechanics as well as in sensing and hand programming. Salisbury [21] studied the mechanics of finger tip grasps under the assumptions of rigid body kinematics and coulomb friction. For an object held in tip prehension, he developed a method to control the hand to impart small arbitrary motions and apply arbitrary forces to the object. Salisbury also developed a method to control the effective Cartesian stiffness of the grasped object. Cutkosky [3] included the effects of the curvature of the finger tips and the structural stiffness of the fingers. Central to Salisbury's formulation is the hand's ability to apply normal forces to the object which are large enough to prevent slipping between the object and any finger. Since slipping cannot always be prevented, Holzmann [8] developed a method to predict slipping and the accompanying friction forces.

There are an infinite number of possible grasps of an object. Jameson [9] applied numerical optimization techniques to choose a three-point grasp which provided complete rigid restraint of the object relative to the hand. Hanafusa and Asada [6] developed a hand with flexible fingers to pick up planar objects. They derived stability conditions and grasp selection criteria based on minimizing the potential energy of the fingers. Others have applied optimization techniques to various objective functions to choose "optimal" grasps $[1,7,9,12,17,22\}$.

The only work to date on planning is for grippers with prismatic joints. Laugier and Wolter $[14,25]$ both plan grasps by considering the volume swept out by a parallel-jawed gripper in its approach to an object. Juan [10] built an interactive system, PAAR, to aid planning assembly tasks. Extending Mason's [15] work in manipulation, Brost [2] developed a technique for planning grasps of polygons which were free to slide on a supporting plane.

The most mathematically complex aspect of grasping is in the manipulation of the grasped object over a large range. Okada [18] programmed a hand with three fingers and eleven degrees of freedom to turn a nut onto a bolt. Such successes are few because the equations describing manipulation are nonlinear, time varying and constrained [11].

\subsection{Problem Statement}

The problem addressed here is that of picking up an object with an articulated mechanical hand in the absence of friction. It has been shown by Lakshminarayana [13] that if a frictionless grasp is used to completely restrain an object, using only finger tips, a hand would need a minimum of seven fingers (four in the twodimensional case). However, the necessary number of fingers may be reduced to three (two in the plane) if the hand's entire palmar surface is used ${ }^{1}$ (this includes the palm and those surfaces of the fingers which face the palm).

\subsection{Assumptions}

A typical two-dimensional "hand" is shown in Figure 1. There are two single-link fingers (bodies 1 and 2 ) and a flat palm. The object (body 5) is initially at rest on the support which is fixed in the world. The $x$ and $y$ axes of the world coordinate frame define the plane of interest. All moments and rotations have nonzero components in the $z$-direction (out of the page). For the mathematical analysis presented in section 2 , we make the following assumptions:

1) the fingers and hand move under exact position control,

2) all bodies are rigid convex polygons,

3) the mass and the position of the center of gravity of each body is known,

4) the kinematic arrangement of the hand is known,

5) the motion proceeds slowly enough to ignore dynamic effects,

6) there are no friction forces acting on the bodies, and

7) the object is initially at rest in a known position on the supporting surface.

As a direct result of the second assumption, we know that for any pair of contacting bodies, the contact occurs either at a point or along a line segment. A line segment contact is treated as two point contacts, one at each end of the segment. This assumption allows for the uniform treatment of all contacts while maintaining the correct kinematic constraints [4].

\footnotetext{
1 This idea was proposed by R. Bajcsy
} 


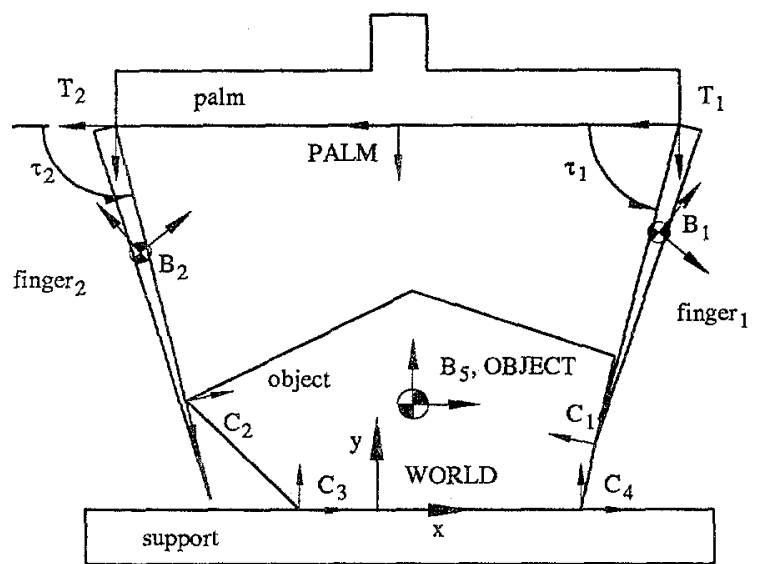

Figure 1: a typical hand in an initial grasping configuration.

\subsection{Notation and Conventions}

The notation and conventions used in this paper are as follows: vectors are indicated by boldface, lower case letters, (e.g. $x$ ), an additional circumflex, $\hat{\mathbf{x}}$, is used to denote unit vectors; matrices are boldface, upper case letters, (e.g. A); a matrix, A, or vector, $\mathbf{x}$ defined with respect to a coordinate frame, $\mathbf{B}$, is written as ${ }^{\mathbf{B}} \mathbf{A}$ or ${ }^{B_{\mathbf{X}}}$, respectively (if $\mathbf{B}$ is the world frame, the superscript is absent); a dot, $\dot{\mathbf{x}}$, over a variable implies its time derivative; $\mathbf{A}^{\mathrm{T}}$, is the transpose of $\mathbf{A}$; and the subscripts, $x, y, z$, when applied to a vector indicate one of its components (e.g. $\mathbf{x}_{x}$ is the $x$-component of the vector, $\mathbf{x}$ ). Bold elements appearing in matrices and vectors denote the submatrices and subvectors of the appropriate dimension. Vector inequalities apply term by term.

\section{Mathematics}

We begin by defining the mathematical framework for the analysis of frictionless grasping in the plane. It is conveninet to define several coordinate frames and represent them as three-bythree homogeneous transformation matrices [19]. Let $C_{i}$ be the contact frame associated with the $i^{\text {th }}$ contact point (see Figure 1). Then

$$
\mathrm{C}_{i}=\left[\begin{array}{ccc}
\hat{\mathbf{n}}_{\mathrm{C}_{i}} & \hat{\mathrm{o}}_{\mathrm{C}_{i}} & \mathrm{p}_{\mathrm{C}_{i}} \\
0 & 0 & 1
\end{array}\right] \quad i=1, \ldots, n_{c}
$$

where $\hat{o}_{C_{i}}$ is the $i^{\text {th }}$ contact normal directed inwardly with respect to the object, $\hat{\mathbf{n}}_{\mathbf{C}_{i}}$ is the contact tangent, $\mathbf{p}_{\mathbf{C}_{i}}$ is the position of the contact, and $n_{c}$ is the number of contact points. There are $n_{b}$ body frames, $\mathbf{B}_{i}$,

$$
\mathbf{B}_{i}=\left[\begin{array}{ccc}
\hat{\mathbf{n}}_{\mathbf{B}_{i}} & \hat{\mathbf{o}}_{\mathbf{B}_{i}} & \mathbf{p}_{\mathbf{B}_{i}} \\
0 & 0 & 1
\end{array}\right] \quad i=1, \ldots, n_{b}
$$

which are fixed to the $i^{\text {th }}$ body, with its origin, $\mathbf{p}_{\mathbf{B}_{i}}$, at the center of mass. At the base joint of the $i^{\text {th }}$ finger, we define $\mathbf{T}_{i}$

$$
\mathbf{T}_{i}=\left[\begin{array}{ccc}
\hat{\mathbf{n}}_{i} & \hat{\mathbf{o}}_{i} & \mathbf{p}_{i} \\
0 & 0 & 1
\end{array}\right] \quad i=1, \ldots, n_{f}
$$

where $n_{f}$ is the number of fingers. The frames' origins are at the centers of the joints about which the actuators apply torques, $\tau_{i} ; i=1, \ldots, n_{f}$.

\subsection{Velocity Constraints}

If there were no contacts between any of the bodies, then the hand and object would be free to move in any manner. However, when executing a grasp, the contacts constrain their motion. Consider two rigid bodies in contact at the point, $\mathbf{p}_{\mathbf{C}_{i}}$, as shown in Figure 2 .

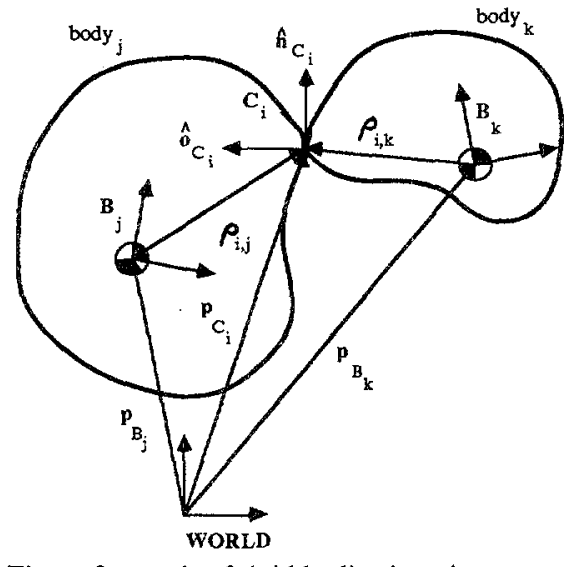

Figure 2: a pair of rigid bodies in point contact.

Let $\mathrm{p}_{i, j}$ be the $i^{\text {th }}$ contact point on the $j^{\text {th }}$ body. The velocity of that contact point on the $j^{\text {th }}$ body is

$$
\dot{\mathbf{p}}_{i, j}=\dot{\mathbf{p}}_{\mathrm{B} j}+\omega_{\mathrm{B} j} \times \rho_{i, j}
$$

where $\dot{\mathbf{p}}_{\mathbf{B}_{i}}$ is the velocity of the center of gravity of the $j^{\text {th }}$ body, $\rho_{i, j}$ is the position of the $i^{\text {th }}$ contact point with respect to the $j^{\text {th }}$ body, and $\omega_{\mathrm{B} j}$ is the angular velocity of the $j^{\text {th }}$ body. Writing equation (4) in matrix form saving only those components relevant to the two-dimensional problem yields

$$
\dot{\mathbf{p}}_{i, j}=\left[\begin{array}{ccc}
1 & 0 & \left(-\rho_{i, j}\right)_{y} \\
0 & 1 & \left(\rho_{i, j}\right)_{x}
\end{array}\right]\left[\begin{array}{c}
\dot{\mathbf{p}}_{\mathbf{B}_{i}} \\
\left|\omega_{\mathbf{B}_{j}}\right|
\end{array}\right]
$$

where $\left|\omega_{\mathbf{B}_{j}}\right|$ is the magnitude of $\omega_{\mathbb{B}_{j}}$. The relative velocity at the contact point is

$$
\mathbf{v}_{i}=\dot{\mathbf{p}}_{i, j}-\dot{\mathbf{p}}_{i, k} \text {. }
$$

Since the bodies slide or roll on one another or separate, the relative velocity constraint imposed by the contact is given by

$$
\hat{\mathbf{o}}_{\mathrm{C}_{i}} \cdot \mathbf{v}_{i} \geq 0 \text {, }
$$

for which the inequality indicates that the objects are separating. If relationship (7) holds as an equality and if in addition $\hat{\mathbf{n}}_{\mathbf{C}_{i}} \cdot \mathbf{v}_{i}=0$, then the bodies are rolling on one another at the contact point; if $\hat{\mathbf{n}}_{\mathrm{C}_{i}} \cdot \mathbf{v}_{i} \neq 0$, then the bodies are sliding.

If we consider the two bodies to be pinned together at the $i^{\text {th }}$ contact point, as is the case for the palm and a finger, it is only possible for the bodies to rotate about the joint. Thus the relative translational velocity at the joint must be zero

$$
\mathbf{v}_{i}=0 \text {. }
$$

(Note that the constraints on the relative motion of two bodies "pinned" at their contact point is identical to the constraints required by rolling contact in planar motion. This however is not the case for three dimensional motion.) Using inequality (7) for each contact point and equation (8) for each finger joint, the system's velocity constraints at a given instant may be written as

$$
\left[\begin{array}{lll}
\mathbf{V}_{\theta} & \mathbf{V}_{o b} & \mathbf{V}_{p}
\end{array}\right]\left[\begin{array}{c}
\dot{\theta} \\
\dot{\mathbf{q}}_{o b} \\
\dot{\mathbf{q}}_{p}
\end{array}\right] \geq\left[\begin{array}{l}
0 \\
0 \\
0
\end{array}\right],
$$

where $\dot{\theta}$ is the vector of joint velocities, $\dot{\mathbf{q}}_{o b}$ is the velocity of the object, and $\dot{q}_{p}$ is the velocity of the palm. It should be noted that the quantity $\mathbf{V}_{o b} \dot{\mathbf{q}}_{o b}$ represents the components of the velocities of the contact points on the object in the directions of their respective contact normals and $\mathbf{V}_{\theta} \dot{\theta}$ and $V_{p} \dot{q}_{p}$ represent the velocities for contact points on the fingers and the palm respectively. 


\subsection{Static Equilibrium}

The hand/object system must satisfy inequality (9) and the equations of static equilibrium. Referring again to Figure 1 , we write

$$
\begin{gathered}
\sum_{i=1}^{n_{c}} \mathbf{f}_{i}=-m_{a b} \mathrm{~g} \\
\sum_{i=1}^{n_{c}} \mathbf{p}_{\mathrm{C}_{i}} \times \mathrm{f}_{i}=-m_{o b} \mathbf{p}_{\mathrm{B}_{\mathrm{s}}} \times \mathbf{g} \\
\tau_{k}+\sum_{i \in \Omega_{k}}-\mathrm{T}^{\mathbf{T}_{k}}\left(\mathbf{p}_{\mathrm{C}_{i}} \times \mathbf{f}_{i}\right)_{z}=-\mathrm{m}_{k} \mathrm{~T}_{k}\left(\mathbf{p}_{\mathrm{B}_{k}} \times \mathbf{g}\right)_{z} ; k=1, \ldots, n_{f}
\end{gathered}
$$

where $\Omega_{k}=\left\{i \mid\right.$ the $i^{\text {th }}$ contact is on the $k^{\text {th }}$ finger $\}, m_{k}$ is the mass of the $k^{\text {th }}$ finger, $m_{o b}$ is the mass of the object, $\mathbf{g}^{\mathrm{T}}=\left[\begin{array}{lll}0 & -\mathrm{g} & 0\end{array}\right]$, and $\mathrm{g}$ is the gravitational acceleration constant. Equations (10) and (11) are the force and moment balance equations, respectively, for the object. Equation (12) is the moment balance for the $k^{\text {th }}$ finger. Writing equations $(10-12)$ in matrix form for the two-dimensional problem gives

$$
\text { where } \mathbf{A}=\left[\begin{array}{ll}
\mathbf{W}_{O} & 0 \\
\mathbf{W}_{F} & \mathbf{I}
\end{array}\right], \quad \mathbf{x}=\left[\begin{array}{l}
\mathbf{c} \\
\tau
\end{array}\right], \quad \mathbf{b}=\left[\begin{array}{c}
\mathbf{w} \\
\mathbf{s}
\end{array}\right],
$$

$\tau$ is the vector of joint moments $\left(n_{f} \times 1\right), \mathrm{c}$ is the vector of contact force magnitudes $\left(n_{c} \times 1\right)$, $\mathbf{w}$ is the vector of gravity forces and moment acting on the object $(3 \times 1), \mathrm{s}$ is the vector of gravity moments acting about the joint axes $\left(n_{f} \times 1\right), \mathrm{I}$ is the identity matrix $\left(n_{f} \times n_{f}\right), 0$ is a zero matrix $\left(3 \times n_{f}\right), \mathbf{W}_{0}$ is the object wrench matrix $\left(3 \times n_{c}\right)$ as defined by Salisbury [16] and $\mathbf{W}_{F}$ is the wrench matrix for the fingers $\left(n_{f} \times n_{c}\right)$. For the definitions of the elements of $\mathbf{W}_{O}, \mathbf{W}_{F}, \mathbf{w}$, and $\mathbf{s}$, see [23].

\subsection{Object Motion}

To plan grasps, we must be able to predict the motion of the object, given the palm and finger motions. If we consider an infinitesimal increment in time, we can rewrite inequalities (9) in terms of differential motions

$$
\mathbf{V}_{o b} \mathbf{d q}_{o b} \geq-\left[\begin{array}{ll}
\mathbf{V}_{\boldsymbol{\theta}} & \mathbf{V}_{p}
\end{array}\right]\left[\begin{array}{c}
\mathbf{d} \boldsymbol{\theta} \\
\mathbf{d q}_{p}
\end{array}\right]
$$

where only $\mathbf{d q}_{o b}$ is unknown. Inequality (14) represents the kinematic constraints on the motion of the object. If we solve for $\mathbf{d q}_{o b}$, we find that the solution is not unique. Figure 3 shows an initial grasping configuration. After moving the fingers together a small amount, the object will move to a new position. Figure 3 illustrates an ambiguity inherent in the kinematic constraints (14). Both Figures $3 \mathrm{~b}$ and $3 \mathrm{c}$ show kinematically admissible solutions, but the position of the object shown in $3 \mathrm{c}$ is physically correct if there is no friction present.
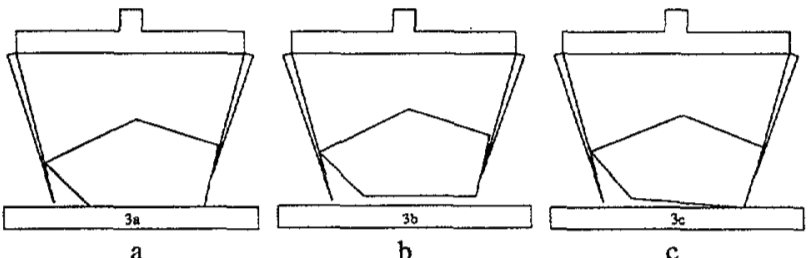

Figure 3: the ambiguity inherent in the kinematic constraints.

We can resolve the kinematic ambiguity by noting that if the motions are slow and there is no friction, then the object must move to minimize its potential energy. Thus the motion is given by the solution to the following linear program
Minimize $\quad \mathrm{y}=\mathrm{m}_{\mathrm{ob}} \gamma^{\top} \dot{\mathrm{q}}_{b b}$

Subject to: $\mathbf{V}_{o b} \dot{\mathbf{q}}_{o b} \geq \xi$

$\dot{q}_{o b}$ unrestricted

where $\gamma=\left[\begin{array}{lll}0 & g & 0\end{array}\right]$ and $\xi=-\left[\begin{array}{ll}V_{\theta} & V_{p}\end{array}\right]\left[\begin{array}{c}\dot{\theta} \\ \dot{q}_{p}\end{array}\right]$

The linear program defined by equations $(15-17)$ is the velocity formulation of the object motion. Its physical interpretation is that the object velocity must satisfy the velocity constraints while minimizing the ouject's rate of potential energy gain. The theory of linear programming provides a dual formulation for every linear program [5]. The dual of the velocity formulation is the force formulation.

$$
\begin{gathered}
\text { Maximize } \quad \mathrm{z}=-\xi^{\mathrm{T}} \lambda \\
\text { Subject to: } \quad \mathrm{V}_{o b}^{\mathrm{T}} \lambda=m_{o b} \gamma \\
\lambda \geq 0
\end{gathered}
$$

The variable, $\lambda$, of the force formulation is the dual variable of the velocity formulation and is the vector of Lagrange multipliers associated with the velocity constraints. The elements of $\lambda$ are also known to be the magnitudes of the contact forces [24] which were denoted earlier as c. Note that the objective function of the force formulation is the virtual work done by the object on the hand and that constraints (19) are the equations of equilibrium of the object taken with respect to its center of mass. Thus, the object's motion is determined by minimizing the virtual work performed on the object while maintaining static equilibrium with nonnegative contact forces (constraints (20)).

\subsection{Grasp Stability}

A frictionless grasp begins as shown in Figure 3a with the object on its support and the hand just touching it. Our goal is to achieve an enveloping grasp of the object. An enveloping grasp is characterized by the object's being completely restrained with respect to the hand when the finger joints are locked. This type of grasp has been termed a form closure grasp (see Figure 4) by Lakshminarayana [13]. Letting $\mathbf{d q}_{p}=0$ and $\mathbf{d} \theta=0$, in inequality (14), we see that for a form closure grasp, the only solution to

$$
v_{o b} \mathbf{d q}_{o b} \geq 0
$$

must be $\mathbf{d q} q_{o b}=0$.

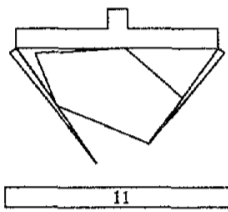

Figure 4: a form closure grasp of the object. If the fingers are locked the object cannot move at all.

Form closure is equivalent to requiring that the contact forces may be combined to resist any applied force and moment. This condition guarantees that the object is always held in equilibrium. The partition of equation (13) which represents the equilibrium of the object is

$$
W_{O} \mathbf{c}=\mathbf{w}^{\prime}
$$

where $w^{\prime}$ may be any disturbing force. Also the contact forces must be compressive, not tensile, so

$$
c \geq 0
$$

Since $w^{\prime}$ is completely free, equations (22) and (23) imply that any vector in Euclidean 3-space, $R^{3}$, can be represented by a nonnegative linear combination of the columns of $\mathbf{W}_{o}$. In other words, the nonnegative column span of $\mathrm{W}_{O}$ must be equivalent to $R^{3}$. This is 
why at least four contact points are needed for a form closure grasp (see [22], Appendix A, pp. 29,30 for a proof).

Let $\mathbf{y}_{i}, i=1, \ldots, n_{c}$ be the columns of $\mathrm{W}_{O}$. The nonnegative span of $\mathbf{y}_{i}$ is given by $C^{+}=\left\{\mathbf{u} \mid \mathbf{u}=\sum_{i=1}^{n_{c}} \alpha_{i} \mathbf{y}_{i} ; \alpha_{i} \geq 0\right.$ for all $\left.i\right\}$. Between the initial state (Figure 1) and the first time that the object is in a form closure grasp, the object is, in general, in equilibrium through force closure [20]. A force closure grasp occurs when $C^{+} \subset R^{3}$ and $w^{\prime} \in C^{+}$. In other words, the object is in equilibrium because the gravity force holds it against the contact points (see Figure 5). If gravity were acting in the opposite direction, then the object would be unstable.

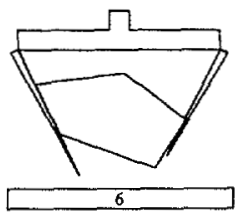

Figure 5: a typical force closure grasp.

In some cases $\mathbf{w}^{\prime} \notin C^{+}$, so the object becomes unstable and falls until a new set of contacts give rise to a new $C^{+}$which contains $w^{\prime}$. This condition usually arises when the object has three contacts and one of the contact forces goes to zero during manipulation.

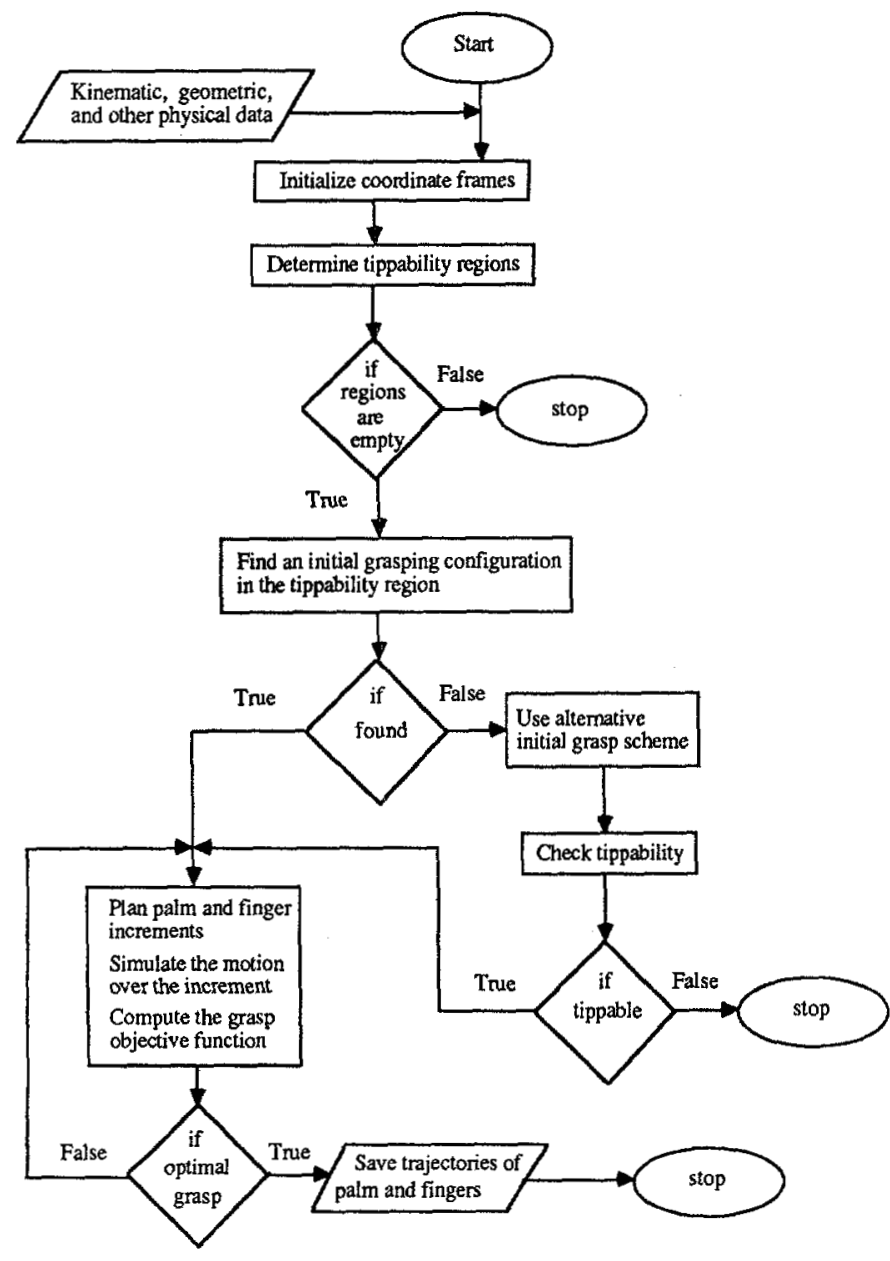

Figure 6: flow chart for Planner/Simulator.

\section{The Planner/Simulator}

The mathematics given above in section 2 have been used to develop a planner/simulator for generating enveloping grasps in the plane. The flow chart (see Figure 6) shows the input to be the kinematic, geometric, and other physical data of the hand, object, and support. After reading the data, all of the coordinate transforms are initialized. Next an initial grasp must be chosen which allows the hand to tip the object. If such a grasp exists, the object is said to be tippable [23].

Definition: An object is tippable if there exist finger contact points on its perimeter (excluding the supporting edge) such that increasing the contact forces applied to those points drives at least one of the supporting contact forces (shown on figure 7 as $f_{3}$ and $f_{4}$ ) to zero.

Figure 7 shows a tippable object and its tippability regions. Assume that one finger tip is placed against the object at the arrowhead. The tippability regions are shown by the curve offset from the left side of the object. The region drawn with a bold solid line indicates the contact positions for the other finger for which squeezing causes vertex 3 to rise. The region traced by the thin solid line causes vertex 4 to move up. The region defined by the dotted line is undesireable for contact because neither the $3^{\text {rd }}$ not the $4^{\text {th }}$ vertex will rise; the object will be pressed against the support. If the curve

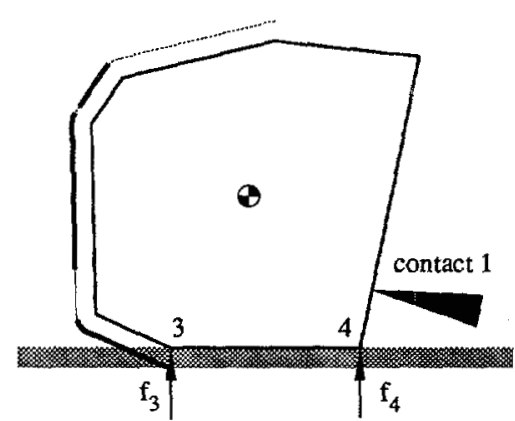

Figure 7: tippability regions of an object.

has no solid portions, then the object cannot be lifted and the program exits. If the object is tippable, then the planner attempts to find an initial grasping configuration in one of the solid regions. If no configuration can be found for which the object will tip, the program exits.

If an initial configuration for the hand is found from which the object can be tipped, the planner determines displacement increments for the hand: palm position and orientation changes and finger angle increments. These increments are sent to the simulation module which computes the resulting motion of the object. The configuration of the hand and object are then updated and sent to the planner for it to plan the next increments. When the planner determines that a suitable grasp has been achieved, it outputs the trajectory and stops.

\section{Results}

The planner/simulator discussed above has been implemented in Fortran 77 using International Mathematics and Statistics Library routines for the solution of systems of nonlinear equations, the solution of linear programs, and the inversion of general matrices. Figure 8 shows a sequence of 10 frames of an object being grasped. The important dimensionless parameters of some of the bodies relative to the palm's width were: the length of finger $1=.6$, and the length of finger $2=7$. The masses of the fingers and the object were proportional to their respective areas and the joint torque limits were chosen to ensure that they would not limit the hand's ability to pick up the object. Planning for this grasp was very simple. First, the palm was centered over the object, parallel to the support, 
just high enough so that the longer finger could not touch the support. Then the palm position was fixed for the rest of the grasping sequence. Next, the fingers were brought into contact with the object after which the fingers were squeezed together at equal angular rates, until a form closure grasp was achieved at frame 11 . Note
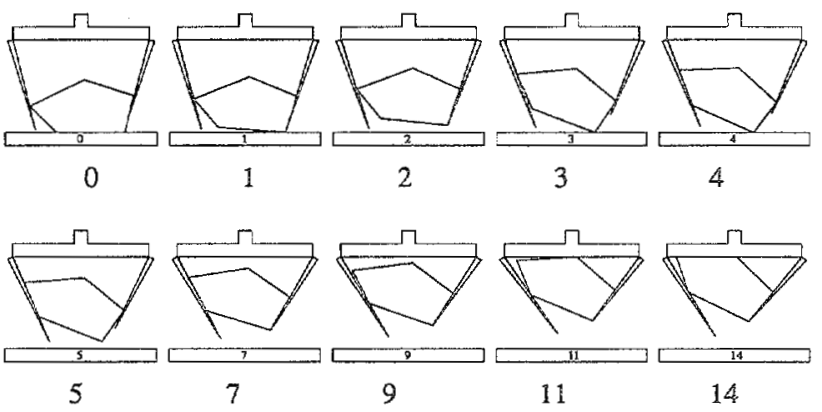

Figure 8: achieving an enveloping grasp.

that during the grasping manipulation, the object moves toward the palm in several different three-contact, force closure grasps. Transitions between the grasps occur when a fourth contact becomes active, forcing one of the previously active contacts to break (see Figure 8 frames $3,4,5$ ). Once the force closure grasp became unstable because one of the contact forces went to zero. In this case, the object executed a constrained fall until another contact stabilized the grasp again (see Figure 8, frame 2,3,4). Frames 12 to 14 were generated by adjusting the grip to minimize the grip objective function (the sum of the contact force magnitudes acting on the object due to gravity loading).

The final grasp, shown in frame 14, has five contact points which cause a discontinuous branch point in the objective function. The branches occur because only four contact points can be maintained while manipulating the grasp, but 5 possible sets of four contacts exist. Two of the five sets do not maintain form closure, so they are not considered for further grip adjustments. Figure 9 shows the grip adjustment objective function in the neighborhood of frame 14.

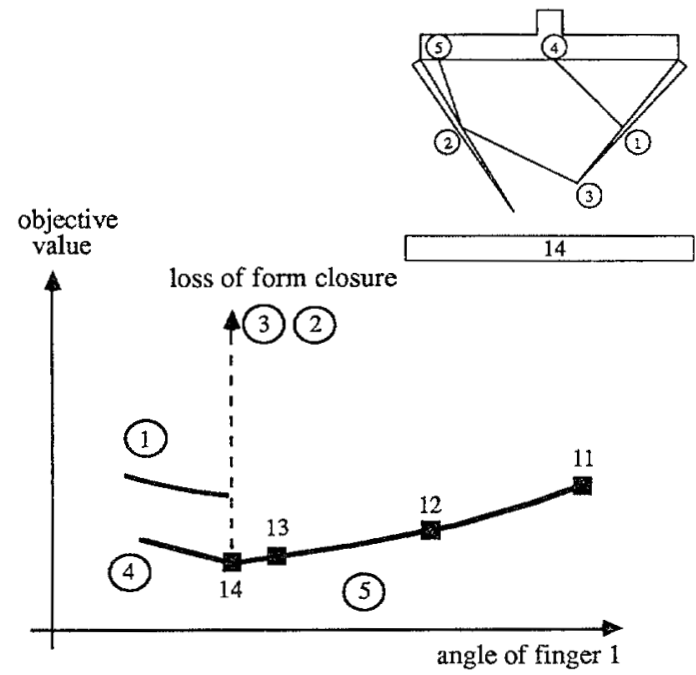

Figure 9: the grip adjustment objective as a function of the angle of finger 1 and the choice of the contact to break. The frame numbers are indicated by squares on the right branch. Each branch is caused by manipulating the object to break one contact and maintain the other four. The circled number beside each branch indicates the lost contact leading to the branch.
The square on the far right corresponds to frame 11. Moving to the left, the objective value decreases until the object gains the fifth contact point at frame 14. To continue manipulating the grip, one of the contacts must be chosen as the one to break, and the finger motions must be computed to be consistent with maintaining the other four contacts. If either contact 2 or 3 is broken, the grasp loses form closure. This is fairly obvious for 2 , since it corresponds to removing the second finger. In the case of the $3^{\text {r }} d$ contact, form closure is lost since the object may rotate counterclockwise about the intersection of the $1^{s t}$ and $4^{t h}$ contact normals, losing contact at points 2 and 5 . If contact 1 is broken, the objective value jumps because one of the palm contact forces (which was previously zero) becomes nonzero, effectively adding to the gravity load which must be absorbed by the two contacts on the fingers. Breaking the $5^{\text {th }}$ contact, the object moves back to a previous grasping configuration. Removing the $4^{\text {th }}$ contact is the only possibility for improving the grasp. However, it is found that the grip objective increases for the manipulation which breaks that contact, so frame 14 is the (locally) optimal solution. The plan is complete.

\section{Conclusion}

A system has been developed which can be used to plan (in an off-line mode) and simulate the grasping of convex polygons when friction and dynamic effects are negligible. In the simulation, the grasps are executed under position control with no need for force control or tactile sensing. However, we have assumed that accurate descriptions of the important physical parameters of the bodies are available to the system.

The mathematics of frictionless grasping is more general than the software implementation described above. Three-dimensional, nonpolyhedral and nonconver bodies may be used as well as multilink fingers. The cost of generality is added computational complexity. The collisions become more difficult to detect, and the constraint equations are more difficult to solve. In addition, the representation of bodies becomes more complex.

In grasp analysis and planning, it is often assumed that the friction forces will be sufficient to prevent any sliding. This is not always the case. Considering the physical limits of any hand and the mass and strength of any object, there is always some limiting value of the coefficient of friction, $\mu$, below which the object will slip. It is less obvious, but equally possible, that the grasping technique described in this paper may fail when the coefficient of friction exceeds some (hand and object dependent) limit. These limits will be explored to define the realms of friction grasping and frictionless grasping, so that the appropriate grasping theory may be applied to any given problem

\section{Acknowledgements}

The authors would like to extend thanks to professor M. Mintz for his invaluable suggestions.

This research was supported by the following grants: IBM 628270, ARO DAA6-29-84-k-0061, AfOSR 82-NM-299, NSF ECS 8411879, NSF MCS-8219196-CER, NSF MCS 82-07294, AVRO DAABO7-84-K-FO77, and NIH 1-RO1-HL-29985-01. Any opinions, findings, conclusions, or recommendations expressed in this publication are those of the authors and do not necessarily reflect the views of the granting agencies.

\section{References}

1. Boissonnat, J. D., "Stable Matching Between a Hand Structure and an Object Silhouette," IEEE Transactions on Pattern Analysis and Machine Intelligence, vol. PAMI-4 no. 6, pp. 603-612, November 1982.

2. Brost, R. C., "Planning Robot Grasping Motions in the Presence of Uncertainty," Carnegie-Mellon Univ. eech. report, vol. CMU-RI-TR-85-12, Pittsburgh, PA, 7/85. 
3. Cutkosky, M. R., in Robotic Grasping and Fine Manipulation, Kluwer Academic Publishers, Boston, 1985.

4. Featherstone, R., "The Dynamics of Rigid Body Systems with Multiple Concurrent Contacts," Proc. of the 3rd ISRR, pp. 24-31, Gouvieux, France, October 7-11, 1985.

5. Gill, P. E., Murray, W., and Wright, M. H., in Practical Optimization, Academic Press, New York, NY, 1981.

6. Hanafusa, H. and Asada, H., "A Robot Hand with Elastic Fingers and its Application to Assembly Process," in Robot Motion, ed. M. Brady, et al., pp. 337-359, MT Press, Cambridge, MS, 1982.

7. Hanafusa, H., et al., "Structural Analysis and Robust Prehension of Robotic Hand-Arm System," Proc. of the International Conference on Advanced Robotics, pp. 311-318, Tokyo, Japan, 1985.

8. Holzmann, W. and McCarthy, J. M., "Computing the Friction Forces Associated with a Three-Fingered Grip," IEEE International Conference on Robotics and Automation, pp. 594 600 , St. Louis, March 18-21, 1985.

9. Jameson, J. W., "Analytic Techniques for Automated Grasping," Ph. D. dissertation, Stanford University, June 1985.

10. Juan, J. and Paul, R. P., Planning Automatic Assembly for Robots (PAAR), to be published in the proc. IEEE International Conference on Robotics and Automation, April, 1986.

11. Kerr, J. R., "An Analysis of Multi-Fingered Hands," Ph. $D$. dissertation, Stanford University, December 1984.

12. Kobayashi, H., "On the Articulated Hands," Proc. of the 2nd ISRR, pp. 128-135, Kyoto, Japan, August 20-23, 1984.

13. Lakshminarayana, K., "Mechanics of Form Closure," ASME report no. 78-DET-32, 1978.

14. Laugier, C. and Pertin, J., Automatic Grasping: A Case Study in Accessibility Analysis, Toulouse, France, January 1983.
15. Mason, M. T., "Mechanics of Pushing," Proc. of the 2nd ISRR, pp. 73-80, Kyoto, Japan, August 20-23, 1984.

16. Mason, M. T. and Salisbury, J. K. Jr., in Robot Hands and the Mechanics of Manipulation, MIT Press, Cambridge, MA, 1985.

17. Nguyen, V., "The Synthesis of Force Closure Grasps in the Plane," A. I. Memo 861, M.I.T. A.I. Lab, Cambridge MS, September, 1985

18. Okada, T., "Computer Control of Multijointed Finger System for Precise Object-Handling," IEEE Transactions on Systems, Man, and Cybernetics; vol. SMC-12, no. 3, pp. 289-298, May/June 1982.

19. Paul, R. P., in Robot Manipulators: Mathematics, Programming, and Control, MIT Press, Cambridge, MS, 1981.

20. Reuleaux, F., The Kinematics of Machinery, Dover Publications, 1963.

21. Salisbury, J. K., "Kinematic and Force Analysis of Articulated Hands," Ph.D. Dissertation, Department of Computer Science, Stanford University, Stanford, CA, July 1982. Report no. STAN-CS-82-921

22. Trinkle, J. C., "Frictionless Grasping," $M S-C I S-85-46$, GRASP Lab 52, University of PA, Philadelphia, November 23, 1985.

23. Trinkle, J. C., Abel, J. M., and Paul, R. P., "An Investigation of Frictionless, Enveloping Grasping in the Plane," $M S$-CIS86-57, GRASP Lab 70, University of PA, Philadelphia, July 1986.

24. Whittaker, E. T., Analytical Dynamics, Cambridge University Press, Cambridge, 1937

25. Wolter, Jan D., Volz, R. A., and Woo, A. C., Automatic Generation of Gripping Positions, University of Michigan, Ann Arbor, MI, February 1984. 